\title{
Múltiplas hiperintensidades no sistema nervoso central em uma criança com neurofibromatose do tipo 1
}

\author{
Multiple central nervous system hyperintensities in a child with neurofibromatosis type 1
}

\author{
Carla Graziadio ${ }^{1}$, Marina Boff Lorenzen², Felipe Nora de Moraes², Rafael Fabiano M. Rosa ${ }^{3}$, Paulo Ricardo G. Zen ${ }^{4}$, Sérgio Fernando \\ Raupp ${ }^{5}$, Giorgio Adriano Paskulin 6
}

\section{RESUMO}

Objetivo: Relatar o caso de uma criança com neurofibromatose do tipo 1, apresentando o achado ocasional de múltiplas hiperintensidades e de lesão hamartomatosa pré-quiasmática no sistema nervoso central.

Descrição do caso: Menino de tez escura de quatro anos, cujo pai apresentava manchas café-com-leite e história de cirurgia de tumor de ouvido. A criança evoluiu com um adequado desenvolvimento neuropsicomotor, sem crises convulsivas ou déficit de aprendizagem. Ao exame físico, possuía múltiplas manchas café-com-leite e efélides. A avaliação pela ressonância nuclear magnética de encéfalo evidenciou múltiplas imagens nodulares hiperintensas em T2 e Flair, com isossinal em T1, localizadas na substância branca dos hemisférios cerebrais, cerebelo e tronco cerebral. Apresentava ainda lesão hamartomatosa pré-quiasmática. A avaliação eletroencefalográfica e o exame neurológico eram normais.

Comentários: As imagens hiperintensas verificadas na ressonância nuclear magnética correspondem aos chamados unidentified bright objects (UBOs), observados em de 60 a $70 \%$ das crianças com neurofibromatose do tipo 1, sendo raros em adultos. Sua etiologia e significado clínico ainda não são cla- ros. Alguns estudos os têm associado à disfunção cognitiva, déficit neurológico e risco de malignização. Hamartomas localizados próximos ao quiasma óptico são incomuns, mas podem se relacionar à puberdade precoce. A avaliação do sistema nervoso central de crianças assintomáticas com neurofibromatose do tipo 1 é discutível. Contudo, nos casos em que UBOs sejam identificados, é importante considerar seu seguimento radiológico, especialmente diante de lesões múltiplas ou atípicas.

Palavras-chave: neurofibromatose 1; manchas cafécom-leite; sistema nervoso central; imagem por ressonância magnética; hamartoma.

\section{ABSTRACT}

Objective: To report a child with neurofibromatosis type 1 presenting the occasional central nervous system feature of multiple hyperintensities and a prechiasmatic hamartomatous lesion.

Case description: The patient is a four-year old black boy whose father presented cafe-au-lait spots and history of ear tumor surgery. The neuropsychomotor development of the child was within the normal range, without seizures
Instituição: Universidade Federal de Ciências da Saúde de Porto Alegre (UFCSPA) e Complexo Hospitalar Santa Casa de Porto Alegre (CHSCPA). 'Doutoranda pelo Programa de Pós-Graduação em Patologia da UFCSPA; Professora da Disciplina de Genética Clínica da UFCSPA, Porto Alegre, RS, Brasil

${ }^{2}$ Graduando do Curso de Medicina da UFCSPA, Porto Alegre, RS, Brasil ${ }^{3}$ Doutorando pelo Programa de Pós-Graduação em Patologia da UFCSPA; Geneticista Clínico do Hospital Materno Infantil Presidente Vargas (HMIPV), Porto Alegre, RS, Brasil

${ }^{4}$ Doutor pelo Programa de Pós-Graduação em Patologia da UFCSPA; Professor da Disciplina de Genética Clínica e do Programa de Pós-Graduação em Patologia da UFCSPA, Porto Alegre, RS, Brasil

${ }^{5}$ Doutor pelo Programa de Pós-Graduação em Medicina da Universidade Federal do Rio Grande do Sul (UFRGS); Neurorradiologista do Hospital São José/Complexo Hospitalar Santa Casa de Porto Alegre (CHSCPA), Porto Alegre, RS, Brasil

${ }^{6}$ Doutor pelo Programa de Pós-Graduação em Genética e Biologia Molecular da UFRGS; Professor da Disciplina de Genética Clínica e do Programa de Pós-Graduação da UFCSPA, Porto Alegre, RS, Brasil
Endereço para correspondência:

Giorgio Adriano Paskulin

Rua Sarmento Leite, 245, sala 403 - Centro

CEP 90050-170 - Porto Alegre/RS

E-mail: paskulin@ufcspa.edu.br

Conflito de interesses: nada a declarar

Recebido em: 24/6/2010

Aprovado em: 26/1/2011 
or learning deficit. At the physical exam, he had several cafe-au-lait spots and freckles. The magnetic resonance image showed multiple nodular and hyperintense images in $\mathrm{T} 2$ and Flair, with isosignal in T1, localized in the white matter of cerebellum, brainstem and cerebral hemispheres. A prechiasmatic hamartomatous lesion was also noted. Electroencephalographic evaluation and the neurological exam were normal.

Comments: The hyperintensities images verified in the magnetic resonance image corresponded to the "unidentified bright objects" (UBOs). They are observed in 60 to $70 \%$ of the children with neurofibromatosis type 1 , being rare in adults. Its etiology and clinical meaning are still not clear. Some studies have associated them to cognitive dysfunction, neurological deficit and risk of malignization. Hamartomas close to the optic chiasm are unusual, but they can be related to early puberty. Central nervous system imaging of asymptomatic neurofibromatosis type 1 children is controversial. However, in cases in which UBOs are occasionally identified, it is important to consider their radiological follow-up, especially in the presence of multiple and atypical lesions.

Key-words: neurofibromatosis 1; cafe-au-lait spots; central nervous system; magnetic resonance imaging; hamartoma.

\section{Introdução}

A neurofibromatose do tipo 1 (NF1 - OMIM 162200)(1) é uma das doenças autossômicas dominantes mais frequentes, com uma prevalência que varia de 1:2.000 a 1:7.800 nascidos vivos. Ela ocorre devido a mutações no gene NF1, localizado na região 17q11.2. A NF1 é considerada uma condição crônica e progressiva, caracterizada por múltiplas manchas café-com-leite, efélides axilares e inguinais, neurofibromas de pele e nódulos de Lisch na íris. Essas manifestações são usualmente encontradas em mais de $90 \%$ dos pacientes afetados ${ }^{(2-7)}$. Entretanto, sua expressividade pode ser extremamente variável, ainda que na mesma família ${ }^{(2,3)}$. A NF1 é considerada também uma doença predisponente ao câncer, uma vez que suas alterações agem em várias vias de sinalização que afetam diferentes processos morfogenéticos e a proliferação celular ${ }^{(8)}$.

Anormalidades do sistema nervoso central (SNC) são comuns e incluem um amplo espectro de alterações que variam de tumores benignos a neoplasias altamente agressivas ${ }^{(3)}$. Dentre essas alterações, destacam-se os gliomas de via óptica.
Embora esses tumores possam causar problemas oftalmológicos, neurológicos ou endócrinos, eles usualmente não produzem sintomas clínicos e são caracterizados por uma progressão bastante lenta ${ }^{(9)}$.

Relata-se aqui o caso de uma criança com NF1 com o achado ocasional do SNC de múltiplas hiperintensidades e uma lesão hamartomatosa pré-quiasmática, discutindo-se os possíveis significados clínicos dessas anormalidades. Este trabalho foi aprovado pelo Comitê de Ética da instituição.

\section{Descrição do caso}

O paciente era um menino de tez escura de quatro anos de idade, o segundo filho de um casal de pais jovens e não consanguíneos. O pai apresentava manchas café-com-leite e história de cirurgia de um tumor de ouvido. A criança nasceu a termo, de parto vaginal, apresentação cefálica, com peso de $3030 \mathrm{~g}$ e Apgar de sete no primeiro minuto. O paciente apresentou desconforto respiratório logo após o nascimento, necessitando ficar hospitalizado durante 11 dias. A mãe referiu que a criança já apresentava manchas café-com-leite ao nascer e estas aumentaram em número durante o crescimento (Figura 1). Seu desenvolvimento neuropsicomotor foi dentro da normalidade.

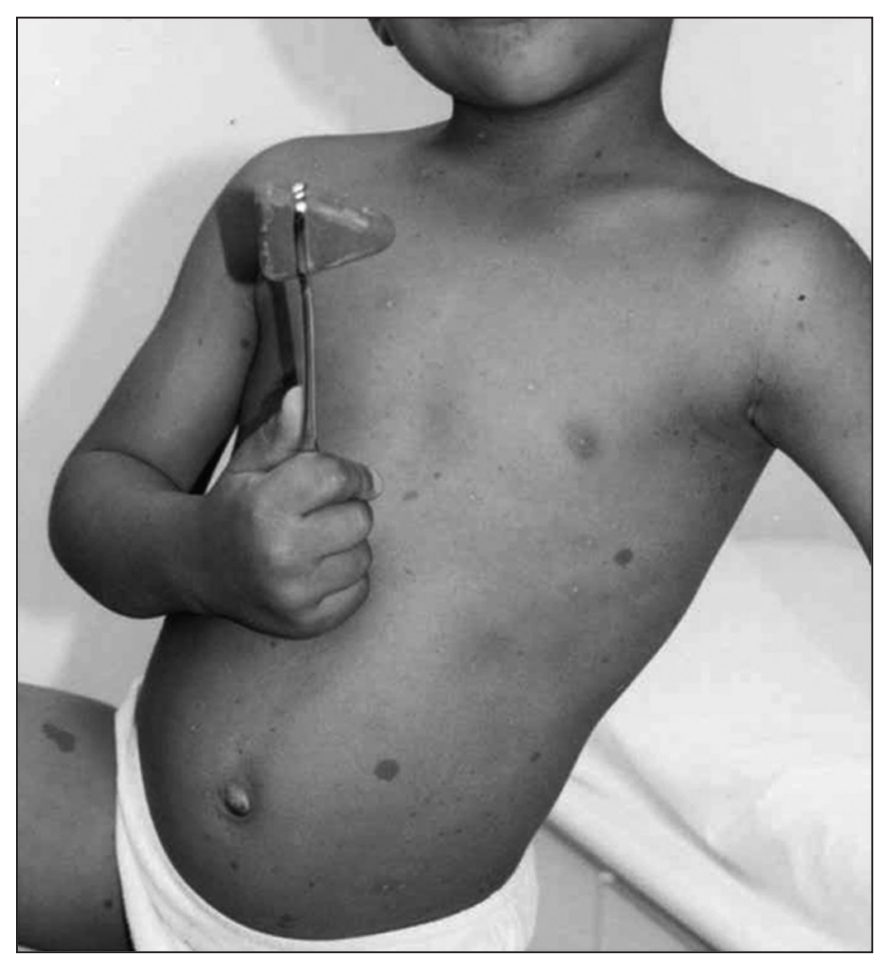

Figura 1 - Pacientes aos cinco anos de idade. Notar as manchas café-com-leite no tronco e nas regiões proximais dos membros, além das efélides no pescoço, axila e abdome 
Aos quatro anos, foi realizada a ressonância nuclear magnética (RNM) do encéfalo devido à suspeita clínica de neurofibromatose. O exame mostrou a presença de múltiplas imagens nodulares e hiperintensas em T2 e Flair, com isossinal em T1, localizadas na substância branca do cerebelo, tronco cerebral e hemisférios cerebrais (Figura 2). Uma lesão hamartomatosa pré-quiasmática também foi verificada (Figura 2). O sistema ventricular mostrava-se de aspecto normal. O paciente possuía medidas antropométricas normais, pesando $20 \mathrm{~kg}$, medindo
$104 \mathrm{~cm}$ e apresentando perímetro cefálico de $53 \mathrm{~cm}$. Ao exame, observou-se: palato alto, múltiplas manchas cafécom-leite (várias maiores do que $5 \mathrm{~mm}$, principalmente no tronco e na região proximal dos membros) e efélides cervicais, axilares e inguinais. Ele não apresentava neurofibromas ou qualquer sinal de puberdade precoce. A avaliação eletroencefalográfica e neurológica eram normais. $\mathrm{Na}$ última avaliação, aos oito anos, possuía sintomas de hiperatividade, entretanto, crises convulsivas ou dificuldades de aprendizagem não foram observadas.
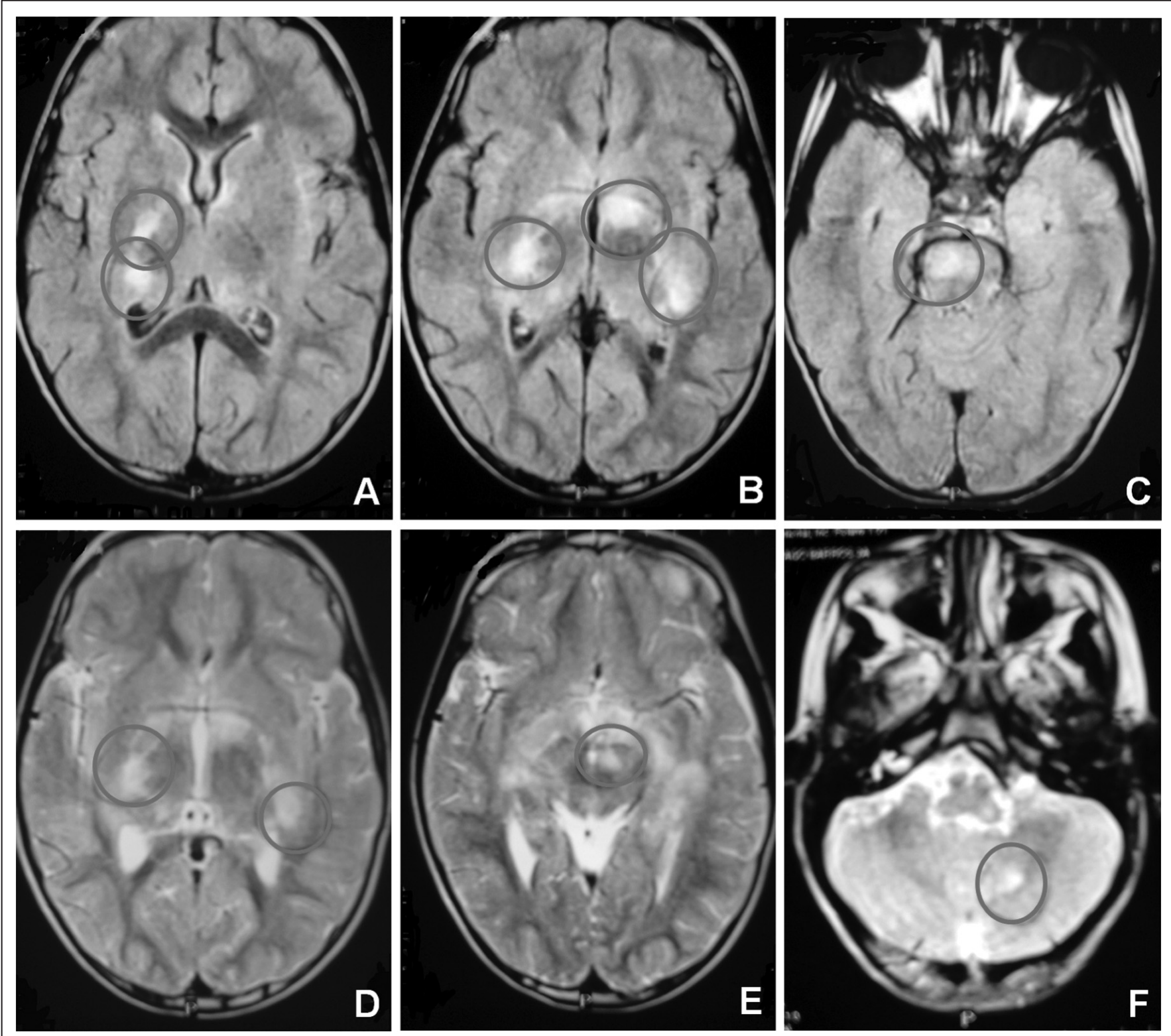

Figura 2 - Ressonância magnética de encéfalo mostrando múltiplas imagens nodulares hiperintensas no Flair (A a C) e em T2 (D a F). Notar a lesão hamartomatosa pré-quiasmática em C 


\section{Discussão}

O diagnóstico de NF1 usualmente se baseia nos achados clínicos apresentados pelos pacientes, seguindo os critérios do National Institute of Health Consensus ${ }^{(10)}$. A presença no paciente aqui descrito de mais de seis manchas café-comleite maiores do que $5 \mathrm{~mm}$, efélides nas regiões axilar e inguinal e um parente em primeiro grau com manchas café-com-leite confirmou o diagnóstico ${ }^{(2-5,11)}$. A testagem genética molecular existe, mas, em geral, não é necessária para o diagnóstico ${ }^{(7)}$. As manchas café-com-leite são o achado mais comum na NF1. Elas são usualmente ovais, achatadas, com bordas irregulares e sua cor varia de amarelo a marrom, sendo sua malignização rara. Efélides são manchas menores do que $5 \mathrm{~mm}$, distribuídas especialmente no pescoço e região inframamária em mulheres, representando a segunda manifestação mais frequente na $N F 1^{(2-5,11)}$. A história familiar de NF1 é verificada em cerca de metade dos $\operatorname{casos}^{(2,4,5)}$.

Anormalidades do SNC são comuns na NF1 e representam uma importante causa de morbidade e mortalidade ${ }^{(3,6,11)}$. As imagens nodulares de alto sinal observadas em nosso paciente consistem dos chamados "objetos brilhantes não identificados" (UBOs, do inglês unidentified bright objects). Eles ocorrem em 60 a $70 \%$ das crianças com NF1 e são raros em adultos. Os UBOs são tipicamente visualizados na sequências T2 da RNM de encéfalo e não identificados na sequência T1 e na tomografia computadorizada. Em geral, são encontrados no cerebelo; entretanto, podem estar presentes também nos gânglios da base, tratos ópticos, tálamo, tronco cerebral e hipocampo ${ }^{(2,3,5,6,9)}$. Sua verdadeira natureza permanece incerta; contudo, acredita-se que representem anormalidades vasculares ou espongiose da substância branca ${ }^{(5,11)}$. Devido à sua significante frequência em crianças, alguns autores têm sugerido que esse achado possa ser utilizado como critério diagnóstico para $\mathrm{NF}^{(6,9)}$. A maior incidência em número e volume dos UBOs ocorre entre quatro e dez anos, sendo raros após os 20 anos de idade. Tal regressão foi sugerida devido ao fato de que o edema intramielínico é um fenômeno intermitente e transitório ${ }^{(2,3,5,6)}$.

Quanto ao seu efeito no quadro clínico, este é ainda incerto; em geral os UBOs não têm sido associados à gravidade da doença ou a qualquer déficit focal ${ }^{(2)}$. Entretanto, alguns estudos mostram uma possível associação entre a presença dos UBOs e a disfunção $\operatorname{cognitiva}{ }^{(2-4,6,11-13)}$. Isso poderia acontecer especialmente na presença de envolvimento do tálamo ${ }^{(14-16)}$ e, talvez, se constituísse em um bom preditor de disfunção cognitiva na idade adulta ${ }^{(5,17)}$. Outros trabalhos têm também associado os UBOs a alterações no desempenho da motricidade fina ${ }^{(12)}$. Em nosso paciente, até a idade de oito anos, não foi verificada qualquer disfunção cognitiva ou déficit neurológico.

Alguns autores sugerem que nem todos os UBOs sejam benignos. Tumores que se desenvolvem nas regiões onde essas hiperintensidades são identificadas têm sido descritos. Se os UBOs crescem após os dez anos de idade, um seguimento cuidadoso deve ser realizado. Sinais adicionais de proliferação maligna são efeitos de massa e distúrbio da barreira hematoencefálica. Um aumento do gadolíneo-DTPA na região dos UBOs é, até o momento, o melhor indicador de uma possível neoplasia. É também interessante notar que crianças com tumores cerebrais frequentemente apresentam um número maior de lesões brilhantes. Assim, recomenda-se realizar o seguimento com RNM com contraste em crianças com UBOs a cada 12 meses $^{(3)}$.

Hamartomas do SNC são comuns em pacientes com NF1 e envolvem especialmente o quiasma óptico. Lesões próximas a essa estrutura, como observado no presente caso, são incomuns e podem levar ao desenvolvimento de puberdade precoce $^{(4,5,11,18)}$. Apesar disso, até a idade de oito anos, o paciente não mostrou qualquer sinal de desenvolvimento sexual.

Existem fortes evidências na literatura sugerindo uma associação entre o transtorno de déficit de atenção e hiperatividade (TDAH) e NF1. Estima-se que a frequência de TDAH em crianças com NF1 seja tão alta quanto $30 \%$. No entanto, há grande escassez de estudos investigando tal relação ${ }^{(19)}$.

Assim, a triagem de anormalidades cerebrais em crianças assintomáticas com NF1 ainda se encontra em discussão e as recomendações existentes são controversas. Em casos com sintomas neurológicos, a RNM de encéfalo é mandatória. Se UBOs são ocasionalmente identificados, é importante considerar o seguimento radiológico do paciente, especialmente na presença de hiperintensidades múltiplas ou atípicas. 


\section{Referências bibliográficas}

1. Online Mendelian Inheritance in Man, OMIM (TM) [homepage on the Internet]. Baltimore e Bethesda: BeMcKusick-Nathans Institute for Genetic Medicine, Johns Hopkins University and National Center for Biotechnology Information, National Library of Medicine [cited 2010 June 15]. Available from: http://www. ncbi.nlm.nih.gov/omim/

2. Lynch TM, Gutmann DH. Neurofibromatosis 1. Neurol Clin N Am 2002;20: 841-65.

3. Mentzel H-J, Seidel J, Fitzek C, Eichhorn A, Vogt S, Reichenbach JR et al. Pediatric brain MRI in neurofibromatosis type I. Eur Radiol 2005;15:814-22.

4. McClatchey Al. Neurofibromatosis. Annu Rev Pathol Mech Dis 2007; 2:191-216.

5. Darrigo-Júnior LG, Bonalumi Filho A, D’Alessandro DS, Geller M. Neurofibromatose tipo 1 na infância: revisão dos aspectos clínicos. Rev Paul Pediatr 2008;26:176-82.

6. Ferraz-Filho JR, Munis MP, Souza AS, Sanches RA, Goloni-Bertollo EM, Pavarino-Bertelli EC. Unidentified bright objects on brain MRI in children as a diagnostic criterion for neurofibromatosis type 1. Pediatr Radiol 2008;38: 305-10.

7. Jett K, Friedman JM. Clinical and genetic aspects of neurofibromatosis 1 . Genet Med 2010;12:1-11.

8. Larizza L, Gervasini C, Natacci F, Riva P. Developmental abnormalities and cancer predisposition in neurofibromatosis type 1. Curr Mol Med 2009;9: 634-53.

9. Rosenbaum T, Engelbrecht V, Krölls W, van Dorsten FA, Hoehn-Berlage M, Lenard HG. MRI abnormalities in neurofibromatosis type 1 (NF1): a study of men and mice. Brain Dev 1999;21:268-73.

10. Neurofibromatosis. Conference statement. National institute of health consensus development conference. Arch Neurol 1988;45:575-8.
11. Kandt RS. Tuberous sclerosis complex and neurofibromatosis type 1: the two most common neurocutaneous diseases. Neurol Clin 2002;20:941-64.

12. Feldmann R, Denecke J, Grenzebach M, Schuierer G, Weglage J. Neurofibromatosis type 1: motor and cognitive function and T2-weighed MRI hyperintensities. Neurology 2003;61:1725-8.

13. Goh WH, Khong PL, Leung CS, Wong VC. T2-weighted hyperintensities (unidentified bright objects) in children with neurofibromatosis 1 : their impact on cognitive function. J Child Neurol 2004;19:853-8.

14. Moore BD, Slopis JM, Schomer D, Jackson EF, Levy BM. Neuropsychological significance of areas of high signal intensity on brain MRIs of children with neurofibromatosis. Neurology 1996;46:1660-8.

15. Hyman SL, Gill DS, Shores EA, Steinberg A, North KN. T2 hyperintensities in children with neurofibromatosis type 1 and their relationship to cognitive functioning. J Neurol Neurosurg Psychiatry 2007;78:1088-91.

16. Chabernaud C, Sirinelli D, Barbier C, Cottier JP, Sembely C, Giraudeau B et al. Thalamo-striatal T2-weighted hyperintensities (unidentified bright objects) correlate with cognitive impairments in neurofibromatosis type 1 during childhood. Dev Neuropsychol 2009;34:736-48.

17. Hyman SL, Gill DS, Shores EA, Steiberg A, Joy P, Gibikote SV et al. Natural history of cognitive deficits and their relationship to MRI T2-hyperintesities in NF1. Neurology 2003;60:1139-45.

18. Biswas K, Kapoor A, Jain S, Ammini AC. Hypothalamic hamartoma as a cause of precocious puberty in neurofibromatosis type 1: patient report. J Pediatr Endocrinol Metab 2000;13:443-4.

19. Keyhan N, Minden D, Ickowicz A. Clinical case rounds in child and adolescent psychiatry: neurofibromatosis type 1 , cognitive impairment, and attention deficit hyperactivity disorder. J Can Acad Child Adolesc Psychiatry 2006;15:87-90. 